\title{
A MONOTONICITY PROPERTY INVOLVING THE GENERALIZED ELLIPTIC INTEGRAL OF THE FIRST KIND
}

\author{
Zhen-HANG YANG AND YU-Ming CHU
}

Abstract. In this paper, we prove that the function

$$
r \rightarrow Y(r)=\frac{\mathscr{K}_{a}(r)}{\sin (\pi a) r^{\prime 2} \log \left(e^{R(a) / 2} / r^{\prime}\right)}-\frac{1}{r^{\prime 2}}
$$

is strictly increasing from $(0,1)$ onto $(\pi /[R(a) \sin (\pi a)]-1, a(1-a))$ for all $a \in(0,1 / 2]$, where $r^{\prime}=\sqrt{1-r^{2}}, \mathscr{K}_{a}(r)$ is the generalized elliptic integral of the first kind, $R(a)=-2 \gamma-\psi(a)-$ $\psi(1-a), \psi$ is the classical psi function and $\gamma=0.57721566 \cdots$ is the Euler-Mascheroni constant.

Mathematics subject classification (2010): 33C05, 33B15, $26 \mathrm{D} 15$.

Keywords and phrases: Gaussian hypergeometric function, generalized elliptic integral, gamma function, psi function, monotonicity.

\section{REFERENCES}

[1] J. G. Wendel, Note on the gamma function, Amer. Math. Monthly, 1948, 55, 563-564.

[2] F. QI, Bounds for the ratio of two gamma functions, J. Inequal. Appl., 2010, 2010, Article ID 493058, 84 pages.

[3] G. D. Anderson, M. K. Vamanamurthy and M. Vuorinen, Conformal Invariants, Inequalities, and Quasiconformal Maps, John Wiley \& Sons, New York, 1997.

[4] P. F. BYRD AND M. D. FrIEDMAN, Handbook of Elliptic Integrals for Engineers and Scientists, Springer-Verlag, New York, 1971.

[5] G. D. Anderson, S.-L. Qiu, M. K. Vamanamurthy And M. Vuorinen, Generalized elliptic integrals and modular equations, Pacific J. Math., 2000, 192 (1), 1-37.

[6] B. C. CARLSON AND J. L. GuSTAFSON, Asymptotic approximations for symmetric elliptic integrals, SIAM J. Math. Anal., 1994, 25 (2), 288-303.

[7] G. D. Anderson, M. K. Vamanamurthy and M. Vuorinen, Functional inequalities for hypergeometric functions and complete elliptic integrals, SIAM J. Math., 1993, 23 (2), 512-524.

[8] R. KÜHNAU, A method for testing the positivity of a function, Z. Angew. Math. Mech., 1994, 74 (2), $140-143$.

[9] S.-L. QiU And M. K. VAmanamuRthy, Sharp estimates for complete elliptic integrals, SIAM J. Math. Anal., 1996, 27 (3), 823-834.

[10] H. ALzER, Sharp inequalities for the complete elliptic integral of the first kind, Math. Proc. Cambridge Philos. Soc., 1998, 124 (2), 309-314.

[11] M.-K. WANG, Y.-M. ChU AND S.-L. QIU, Sharp bounds for generalized elliptic integrals of the first kind, J. Math. Anal. Appl., 2015, 429 (2), 744-757.

[12] M.-K. WANG, Y.-M. ChU AND S.-L. QIU, Some monotonicity properties of the generalized elliptic integrals with applications, Math. Inequal. Appl., 2013, 16 (3), 671-677.

[13] M. BIERNACKI AND J. KRZYŻ, On the monotonity of certain functionals in the theory of analytic functions, Ann. Univ. Mariae Curie-Skłodowska. Sect. A, 1955, 9, 135-147.

[14] ZH.-H. YANG, Y.-M. ChU AND M.-K. WANG, Monotonicity criterion for the quotient of power series with applications, J. Math. Anal. Appl., 2015, 428 (1), 587-604. 
[15] ZH.-H. YANG, A new way to prove L'Hospital monotone rules with applications, arXiv:1409.6408 [math.CA], available online at http://arxiv.org/pdf/1409.6408v1.pdf. 